\title{
Aprendizaje basado en el juego aplicado a la arquitectura prehispánica
} enseñanza de la historia de la

\author{
Game based learning applied to Teaching the history of \\ Pre-Hispanic Architecture
}

\author{
Resumen
}

Yenny Andrea Real Ramos* yreal@javeriana.edu.co Juan Guillermo Yunda* yunda.j@javeriana.edu.co

*Pontificia Universidad Javeriana

Colombia

Recibido: $17 / \mathrm{Jul} / 2020$ Aceptado: 18/Nov/2020
C amino al Sol es una estrategia pedagógica para mejorar el aprendizaje del periodo prehispánico de la historia de la arquitectura para estudiantes universitarios. Se basa en la teoría del Aprendizaje Basado en el Juego (ABJ), respondiendo a una desmotivación ante las clases, consideradas por los estudiantes "densas, pasivas y monótonas." Formulamos un proyecto de investigación para implementar el ABJ a través de un juego de mesa destinado a afianzar conocimientos de las características de la arquitectura y el urbanismo de las culturas Maya, Azteca, Inca y Tairona. El estudio de estas culturas es un aspecto clave de le enseñanza de la arquitectura en Latinoamérica. Para entender el aporte de esta nueva estrategia, durante un año realizamos un seguimiento del aprendizaje de los estudiantes analizando sus logros académicos, realizando entrevistas individuales y grupos focales. Encontramos una respuesta positiva de los estudiantes al $A B J$ y un incremento de los puntajes de sus evaluaciones. Esta evidencia demuestra que es posible implementar el $A B J$ en la enseñanza de arquitectura y puede ser una respuesta ante los retos de desinterés y falta de concentración de los estudiantes. También identificamos una relación interesante entre el proceso de aprendizaje en los estudiantes y nuestro proyecto.

Palabras clave: aprendizaje basado en el juego; gamificación; historia de la arquitectura prehispánica; enseñanza; aprendizaje.

\section{Abstract:}

Camino al Sol (road to the sun) is a pedagogical strategy to improve the learning of the pre-Hispanic period of the history of architecture for undergraduate students. It is based on the theory of Game Based Learning (GLA), responding to a lack of motivation towards the classes, considered by students "dense, passive and monotonous." We formulated a research project to implement the ABJ through a board game aimed at strengthening knowledge of the characteristics of architecture and urbanism of the Mayan, Aztec, Inca and Tairona cultures. The study of these cultures is a key aspect of the teaching of architecture in Latin America. To understand the contribution of this new strategy, for one year we monitored student learning by analyzing their academic achievements, conducting individual interviews and focus groups. We found a positive response from students to the $A B J$ and an increase in their assessment scores. This evidence shows that it is possible to implement the $A B J$ in the teaching of architecture and it can be a response to the challenges of disinterest and lack of concentration of students. We also identify an interesting relationship between the learning process in students and our project.

Keywords: game based learning; gamification; prehispanic architecture history; teaching; learning. 


\section{Introducción}

Para los estudiantes de la carrera de Arquitectura de la Pontificia Universidad Javeriana (PUJ) Bogotá, es de primera necesidad el conocimiento histórico de la arquitectura y el urbanismo. El currículo se divide en cuatro contenidos o asignaturas, 1) Historia de la arquitectura y urbanismo en las culturas antiguas; 2) Historia de la arquitectura y urbanismo paleocristiano, bizantino, medieval, renacimiento y barroco; 3 Historia de la arquitectura y urbanismo moderno occidental; y 4) Historia de la arquitectura y urbanismo prehispánico, hispánico y moderno. En esta última asignatura es en la cual se aplica la estrategia del aprendizaje basado en el juego. Uno de los objetivos de la asignatura arquitectura hispanoamericana es identificar las características espaciales, constructivas y tecnológicas presentes en la arquitectura y urbanismo en las culturas de América prehispánica. Como resultado de aprendizaje esperado (RAE), se espera que el estudiante identifique en el tiempo y el espacio americano los lugares donde se establecieron las culturas prehispánicas, y analice las características espaciales, constructivas y tecnológicas presentes en la arquitectura y el urbanismo de las mismas.

La enseñanza de las clases de historia de la arquitectura en la PUJ se basa en un tipo de enseñanza instruccional. El tipo instrucciona se fundamenta en la clase magistral, donde los profesores tienen un papel protagónico, y en ocasiones el estudiante es un espectador pasivo o receptor de información. De esta manera, esta modalidad se fundamenta en datos memorísticos, donde el profesor imparte la clase y los estudiantes son receptores y deben interiorizar y grabarse la información, para luego rendir sus conocimientos en un examen

Por otro lado, en el área de historia, para evaluar los conocimientos aprendidos por los estudiantes, una de las estrategias más recurrentes es la de la evaluación formativa. Según Shepard, "la evaluación formativa puede implicar métodos informales, tales como la observación y las preguntas orales; o el uso formativo de medidas más formales como exámenes tradicionales, portafolios y evaluaciones de desempeño" (citado en $C A E+E, 2016$, p. 49). En la experiencia, como docentes, el recurso más utilizado para evaluar los conocimientos teóricos son los exámenes escritos. Frente a estos, los estudiantes a menudo consideran que estudian para rendir conocimientos acumulados a corto plazo y no quedan experiencias significativas del aprendizaje.

Reconociendo las estrategias y las evaluaciones, que repercuten en la falta de motivación de los estudiantes por entender y aprender historia, surgen las preguntas centrales de la investigación, las cuales son: ¿cómo desarrollar otras formas pedagógicas dentro de una asignatura teórica, para mejorar los niveles de motivación y aprendizaje significativo en los estudiantes? De igual forma, ¿cómo motivar el estudio de las culturas autóctonas de manera que se refuercen identidades y se valore nuestro pasado?

Para resolver estas preguntas, este proyecto retomó la teoría del Aprendizaje Basado en el Juego (ABJ), también conocido en la literatura inglesa como GBL (Game Based Learning), en la cual los juegos crean escenarios propicios para despertar en los estudiantes una mayor motivación en el aprendizaje, además de propiciar una mayor socialización y participación en clase. Asimismo, se incluyeron aspectos de la literatura sobre gamificación. Por lo general, este término hace referencia a los videojuegos, o a gestiones para incrementar la motivación en actividades que se llevan a cabo en entornos no lúdicos. En la segunda sección de este texto se explicará a detalle el $A B J$ y su relación con la enseñanza en el área de historia de la arquitectura; también se discutirán las ideas sobre gamificación que se aplicaron en el ejercicio.

En una tercera sección, se explicará a profundidad e proceso metodológico que tuvo nuestra propuesta; explicaremos su origen y evolución, evidenciando el proceso de diseño y cómo esta experiencia relaciona el proceso de aprendizaje con nuestro proyecto de investigación creación. Como indican Lion y Maggio (2019, p.20), la investigación profundiza en los procesos que resultan de un devenir y toma de decisiones, transformando las prácticas de enseñanza de manera profunda y perdurable.

En la cuarta sección se explica en qué consiste el juego, sus dinámicas, mecánicas y componentes, y su proceso de implementación. En una quinta sección desarrollaremos a profundidad el proceso de seguimiento y validación de la estrategia, a través de datos cualitativos y cuantitativos sobre los resultados de aprendizaje esperados de los estudiantes para validar el aporte real a su formación. Finalmente, expondremos una serie de conclusiones que muestran cómo nuestro proceso puede aportar a la enseñanza de la arquitectura y de otras disciplinas en nuestra institución, o instituciones similares.

\section{Marco teórico: concepto, elementos y aprendizajes del} juego

En la enseñanza de historia, la mayoría de los profesores han abandonado aquellas explicaciones que se limitaban a describir listados de hechos, personajes y fechas históricas, que los alumnos debían memorizar y luego recitar en los exámenes. De esta manera, los juegos de historia educativos pueden ser usados como alternativa para que el aprendizaje de la historia sea una experiencia motivadora (Nor Azan, Azizah y Wong Seng, 2009). El docente diseña las dinámicas, mecánicas y componentes creando escenarios y narrativas para hacer el aprendizaje atractivo, combinando estrategias didácticas en las 
cuales puede usar herramientas tanto físicas como virtuales. Este modelo de aperendizaje debe proveer al jugador retroalimentación inmediata, objetivos claros y retos que estén a nivel de sus habilidades (Kiili, 2005). La investigación empírica ha probado que el uso de juegos en el aula, efectivamente contribuye al aprendizaje de los estudiantes (Prensky, 2003; Hamari et al., 2016; Qian y Clark, 2016).

En la enseñanza tradicional, por el contrario, se utiliza una pedagogía basada en la clase magistral: el profesor enseña contando lo que sabe y los estudiantes escuchan. Posteriormente, el aprendizaje debe producirse o consolidarse a través del estudio fuera de clase. Las prácticas y seminarios sirven para aplicar lo aprendido a posteriori y, finalmente, se realiza un examen en clase para evaluar lo aprendido y calificar el aprendizaje de los alumnos (Prieto Martin, Díaz Martin, Monserrat Sanz y Reyes Martín, 2014)

Sin embargo, para los estudiantes en general es difíci mantener la concentración por largo tiempo durante las clases magistrales, lo que repercute en su capacidad de aprendizaje. Como sustenta Alba Dorado: "El juego nos saca del orden obligado, de percepciones y concepciones habituales; nos abre hacia lo otro, lo inventado, lo imaginado, lo antes imperceptible e inconcebible, y genera un ambiente propicio para el aprendizaje" (2018, p. 20). Por esta razón, la PUJ se ha preocupado por desarrollar y aplicar nuevas metodologías de docencia, entre ellas algunas basadas en ABJ. Ejemplo de esto es el programa "Círculos de innovación", del Centro para e Aprendizaje, la Enseñanza y la Evaluación $(\mathrm{CAE}+\mathrm{E}){ }^{1}$

De acuerdo con Cortizo et al. (2011), Kiili (2005) y Verdú Surroca (2016), los juegos pueden ayudar a fomentar la colaboración mediante el consenso entre los miembros del grupo. Los participantes tienen que desplegar las habilidades de intercambio de ideas, opiniones, conocimientos, y la tolerancia con las ideas de los demás, aparte de la elaboración y aportación de nuevos conocimientos. Esto crea un entorno positivo de negociación de las perspectivas e ideas surgidas y la toma de decisiones. De esta manera, el ABJ tiene puntos a favor, como la retroalimentación en tiempo real, la socialización, el desarrollo de la creatividad y el fomento de la participación. Mientras los estudiantes asimilan, acomodan y requilibran conocimientos, experimentan altos niveles de significatividad, motivación y atención. Este aprendizaje contribuye a que ese conocimiento se quede en la memoria a largo plazo (Verdú Surroca, 2016).

Se puede plantear que el juego, a pesar de ser un concepto antiguo, se puede considerar, además, como un elemento creador en el cual se reflejan las tradiciones culturales de un momento histórico (Pisabarro Marrón y Vivaracho Pascual, 2018). Lo lúdico como aporte a la educación tampoco es nuevo. Los antiguos romanos llamaban a las escuelas de primeras letras "Iudus", cuyo significado es precisamente, juego; y era un "mágister ludi" el maestro que se encargaba de alfabetizar haciéndoles jugar, con letras construidas con marfil y madera. Aprender jugando es una manera placentera, motivadora y eficiente (Oliva, 2016). A partir de estas

\footnotetext{
${ }^{1}$ https://caee.javeriana.edu.co/circulos-innovacion
}

ideas se han desarrollado diferentes teorías acerca de la aplicabilidad del juego al aprendizaje. En este caso, vamos a discutir el Aprendizaje Basado en el Juego y la gamificación.

\subsection{El ABJ y la gamificación}

Generalmente se piensa que el $A B J$ es una técnica para la enseñanza de la educación básica primaria y de preescolar pero difícilmente un docente de hoy cuestionará el valor social del juego en tanto producto cultural e instrumento pedagógico. En consecuencia, todavía se presentan resistencias al momento de utilizar los juegos de manera formal dentro de los procesos educativos, en particular aquellos que conciernen a temas y niveles de formación que la tradición considera "serios", como son las ciencias exactas o naturales y la educación superior (MontoyaÁlvarez y Uribe-Ciro, 2016). No obstante, en los últimos años el $A B J$ ha retomado una nueva vigencia en el marco de los retos actuales en pedagogía. En la actualidad, la disponibilidad de aparatos electrónicos y la conectividad a internet ha dificultado los procesos tradicionales de enseñanza en el aula. Por ejemplo, durante la clase los estudiantes se distraen revisando sus redes sociales o trabajando en actividades ajenas a las asignadas en el aula. Con respecto a esto, consideramos que la tecnología per sé no debe ser un impedimento para el aprendizaje en el aula, sino un aliado para el docente.

EI $A B J$ se origina como una estrategia didáctica para estimular el aprendizaje de los alumnos. Conjuga e aprendizaje con diferentes recursos conocidos como juegos, en particular aquellos digitales o de la naturaleza computacional, con el fin de apoyar y mejorar la enseñanza (Oliva, 2016). Por otro lado, existe el término gamificación, que proviene de la palabra inglesa gamification, que fundamentalmente es la aplicación de conceptos que encontramos habitualmente en los videojuegos, u otro tipo de actividades lúdicas, buscando la motivación o la fidelización de los usuarios (Cortizo et. al., 2011; Plass, Homer y Kinzer, 2015).

La gamificación remite a aquellas iniciativas orientadas a incrementar la motivación de los estudiantes a partir de la propuesta de experiencias de juego en contextos formativos. Estas prácticas propician un entorno favorable para el desarrollo de habilidades y aprendizajes de diverso tipo, minimizando el esfuerzo cognitivo que pudieran conllevar, y sobre todo buscando una mayor implicación de los sujetos a partir de un clima de competitividad y/o cooperación, orientado al logro de objetivos educativos determinados de modo semejante a como lo hacen los videojuegos. (Villalustre Martínez y del Moral Pérez, 2015).

Gamificación no es convertir la clase en juego. Es recurrir a un juego para captar la atención de los estudiantes y volver más atractiva la clase. Contribuye a que durante la clase se fomente la participación, con el propósito de captar su atención e involucrarlos en la dinámica de la clase de forma amena y motivante (Oliva, 2016). En este punto es importante aclarar las diferencias entre $A B J$ y gamificación, y para entenderlas citamos la (Tabla 1) que contrasta los puntos fundamentales entre los dos conceptos. 


\begin{tabular}{|l|l|}
\hline \multicolumn{1}{|c|}{ ABJ } & \multicolumn{1}{c|}{ Gamificación } \\
\hline $\begin{array}{l}\text { Usa (video) juegos con } \\
\text { fines didácticos }\end{array}$ & $\begin{array}{l}\text { Se centra en utilizar mecá- } \\
\text { nicas de (video) juegos en } \\
\text { entornos no lúdicos }\end{array}$ \\
\hline $\begin{array}{l}\text { Los juegos tienen un } \\
\text { objetivo de aprendizaje } \\
\text { específico }\end{array}$ & $\begin{array}{l}\text { Se le pide al participante } \\
\text { cumplir con ciertas tareas } \\
\text { para obtener recompensa }\end{array}$ \\
\hline $\begin{array}{l}\text { La mayoría de las veces, } \\
\text { el solo jugar resulta grati- } \\
\text { ficante }\end{array}$ & $\begin{array}{l}\text { La motivación es entera- } \\
\text { mente opcional }\end{array}$ \\
\hline $\begin{array}{l}\text { Es más complicado y costo- } \\
\text { so de desarrollar }\end{array}$ & $\begin{array}{l}\text { Usualmente es fácil y bara- } \\
\text { to de crear / estructurar }\end{array}$ \\
\hline $\begin{array}{l}\text { El contenido se adapta a la } \\
\text { estructura del juego }\end{array}$ & $\begin{array}{l}\text { Los elementos de un juego } \\
\text { se pueden ensamblar }\end{array}$ \\
\hline $\begin{array}{l}\text { Existe la posibilidad de } \\
\text { perder }\end{array}$ & $\begin{array}{l}\text { Perder no es una opción, el } \\
\text { objetivo es motivar }\end{array}$ \\
\hline
\end{tabular}

Tabla 1: Comparación ABJ y gamificación Fuente: Adaptado de Oliva, 2016, p.44

Por ejemplo, como indica la tabla, en el ABJ los juegos tienen un propósito principalmente pedagógico, mientras la gamificación pude aplicarse en diferentes contextos, no necesariamente didácticos. Un ejemplo de esto puede ser la aplicación Waze, que a través de estrategias de gamificación incentiva a los conductores a compartir con otras personas datos sobre el tráfico en tiempo real. Esto no es un contexto didáctico, ya que el conductor no está esperando obtener un aprendizaje nuevo, sino conocer el estado del tráfico en tiempo real. La aplicación ofrece recompensas de prestigio, al incrementar el nivel y/o puntos.

Igualmente, en el $\mathrm{ABJ}$ el estudiante tiene la opción de perder si no logra cumplir los objetivos del juego, y con esto incumple sus resultados de aprendizaje esperados. Por otro lado, en la gamificación no existe la posibilidad de perder. Retomando el ejemplo anterior, en la aplicación Waze un conductor puede optar por no ganar puntos, y a pesar de esto puede seguir obteniendo información de tráfico. La gamificación, a diferencia del $A B J$, se puede descomponer, mientras una actividad de ABJ está estructurada con un objetivo, un principio y un final; Waze, por ejemplo, tiene diferentes actividades que pueden ser independientes entre sí. Un conductor puede tener una red social de amigos, o crear y personalizar su avatar, o tener rangos, puntos o logros con los cuales compite con otros conductores.

Además de estos dos conceptos, la literatura también menciona una serie de elementos en los cuales podemos descomponer la actividad lúdica o juego. Es importante tener en cuenta estos elementos en el momento de diseñar la actividad del ABJ o gamificada, ya que de lo contrario no se podría calificar como tal.

\subsection{Los elementos del juego}

Según Werbach y Hunter, citados por Pisabarro Marrón y Vivaracho Pascual (2018), los elementos básicos de los juegos son tres, y se estructuran de forma piramidal: las dinámicas en la cima, las mecánicas en el medio y los componentes en la base, como lo muestra la Figura 1. Además de estos elementos, existe un cuarto, fuera de la pirámide rodeándola, que sería la experiencia que se vive en el juego. La suma de estos componentes crea un juego, y estos deben estar integrados para que el juego funcione.

En primer lugar, las dinámicas, se pueden definir como el gran enfoque del juego. Son los aspectos generales que incluyen las limitaciones, las emociones, la narrativa, la progresión y las relaciones que se establecen en el juego. También se pueden incluir en esta categoría los contenidos temáticos, que resumen los resultados de aprendizaje que se esperan del juego. Siguiendo en orden descendente se encuentran las mecánicas que, según las ideas de gamificación, son las reglas propias del juego, lo que permite a los participantes avanzar. Pueden incluir las reglas del juego, mecanismos de control, niveles, sistema de puntos, desafíos, suerte, competencia, retos, realimentación, adquisición de recursos, recompensas, transacciones y turnos. En la base de la pirámide se encuentran los componentes. Estos son los objetos que son diseñados y construidos para llevar a cabo el juego. Pueden incluir avatares, insignias, colecciones, desbloqueo de contenido, regalos, tablero de marcadores, niveles, y puntos.

Finalmente, alrededor se encuentra la experiencia, que puede definirse como las respuestas emocionales durante el desarrollo del juego que lo hacen sentir real. Entre ellas se incluye la diversión.

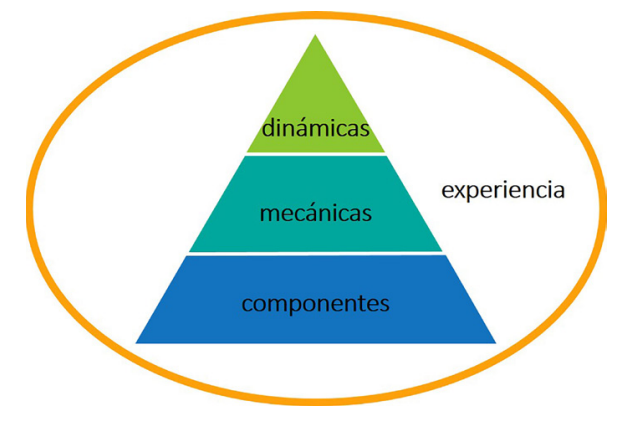

Figura 1: Dimensiones de la gamificación Fuente: Pisabarro Marrón y Vivaracho Pascual, 2018, p.87

Todo lo anterior motivó el desarrollo de Camino al Sol como una metodología de $A B J$, que incorpora estrategias de la gamificación para potenciar la motivación de los estudiantes. A partir de este marco teórico mixto que planteamos para el juego Camino al Sol, en la siguiente sección describiremos nuestro proceso a la luz de los elementos antes mencionados, dinámicas, mecánicas, componentes y la experiencia.

\section{Metodología: Aprendizaje Basado en el Juego a través de Camino al Sol}

Para llegar al prototipo de Camino al Sol, se partió de un proyecto planteado en clase junto con los estudiantes. Como producto de este ejercicio nace la primera versión de juego, llamada "Prehispanópolis", la cual se explicará con mayor detenimiento en el desarrollo de este capítulo, en el numeral 3.1. Esta primera versión se toma 
como base para plantear un proyecto de investigación en donde se hacen ajustes de contenido, de dinámicas, de mecánicas y de componentes, además de unificar e diseño visual para producir el primer modelo ajustado de Camino al Sol, al cual se le hacen los ajustes necesarios tras hacer pruebas y validaciones con profesores y estudiantes. De esta manera se produce el prototipo de Camino al Sol, el cual se expondrá en detalle en el numeral 3.2

\subsection{Primera versión, "Prehispanópolis"}

En las clases de historia de la arquitectura hispanoamericana, desde hace varios años se han venido implementando diferentes cortos ejercicios de docencia para evaluar los conocimientos. Estos ejercicios están relacionados con el ABJ y la gamificación. Del ABJ, juegos elaborados por los estudiantes como crucigramas y escaleras, entre otros, y de la gamificación se utilizan recursos como Kahoot ${ }^{2}$, para evaluar los conocimientos vistos en clase, bien sea impartidos por el docente o expuestos por los estudiantes. Esta herramienta ofrece preguntas de selección múltiple y los resultados se visualizan en un podio que estimula la motivación y competencia de los estudiantes.

En el primer semestre del año 2018, luego de realizar las exposiciones de la arquitectura de principales ciudades prehispánicas, en conjunto con los diecinueve (19) estudiantes se elaboró un juego de mesa llamado Prehispanópolis (Figura 2). Este juego se creó como una nueva forma pedagógica dinámica para el fortalecimiento de los conocimientos adquiridos de arquitectura y urbanismo prehispánico.

El proceso para la elaboración de este juego inició con una lluvia de ideas entre todos los estudiantes para establecer el tipo y las reglas del juego. Después de tener estos puntos claros, para perfeccionar la idea se dividió e grupo en subgrupos y cada uno se encargó de desarrollar una parte del material con unos parámetros de tamaño y diagramación preestablecidos. Por ejemplo, unos realizaron la caja, otros el tablero, las tarjetas, el mapa, las fichas y las instrucciones. La mecánica del juego consistía en lanzar el dado e ir avanzando por el tablero con las fichas, y responder acertadamente a la pregunta de la categoría determinada para poder adquirir la mayor cantidad de propiedades posible y a su vez ocupar los territorios en el mapa.

El desarrollo y elaboración del material fue un proceso de tres semanas. La clase tiene una intensidad horaria de dos horas semanales, y en cada uno de estos tres encuentros se le dedicaba un tiempo al final de la clase para compartir y ajustar los avances que cada grupo había elaborado. Los mismos estudiantes jugaron en el salón de clase (Figura 3) y validaron las dinámicas, las mecánicas, los componentes y la experiencia de Prehispanópolis.

\footnotetext{
2 https://kahoot.com/schools-u/

3 La Pontificia Universidad Javeriana apoya a proyectos de investigación-creación que se originen en una pregunta o problema de investigación y busquen hacer aportes originales desde la creación y la práctica artística, contribuyendo a la generación de conocimiento transferible más allá de su campo respectivo. La propuesta debe conducir a aportes originales en los campos de las artes visuales y escénicas, la música, el diseño, la arquitectura, la literatura o la producción audiovisual.
}

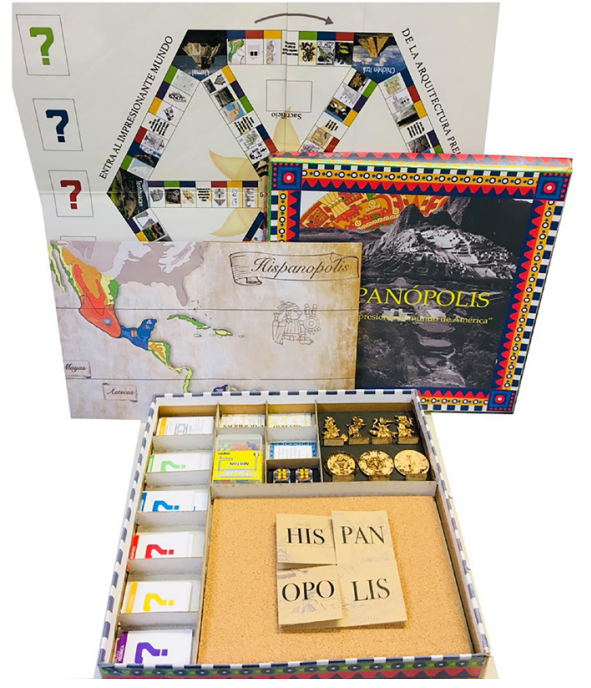

Figura 2: Primera versión Prehispanópolis

Fuente: Elaboración propia

En cuanto a las mecánicas, pese a que se pudo jugar con fluidez, algunas reglas no funcionaban, eran confusas y no era claro cómo proceder en algunos casos. También, algunos tipos de pregunta no eran los más adecuados por ser preguntas abiertas, y no se podía establecer fácilmente si eran válidas para obtener puntos o no. Por último, el uso del mapa, en el cual se ponían las fichas ganadas, no tenía mayor relevancia en el juego. Respecto a los componentes, aunque el resultado visual fue aceptable, faltó una mayor unidad gráfica y algunos materiales no eran muy resistentes a la manipulación propia del juego. Finalmente, en lo concerniente a la experiencia, se valoró muy positivamente por parte de los estudiantes el trabajo en equipo, la satisfacción de ver materializada una idea, el interés por aprender de las culturas prehispánicas, la apropiación del tema a medida que iban elaborando el material y, sobre todo, el aprender de una manera divertida al momento de juagar en la clase.

Esta primera versión de Prehispanópolis sirvió como base de formulación de un proyecto de investigacióncreación ${ }^{3}$ desarrollado durante el año 2019. El objetivo principal de esta investigación era ajustar, rediseñar, probar y validar la nueva versión del juego en cuanto a contenido, jugabilidad y diseño.
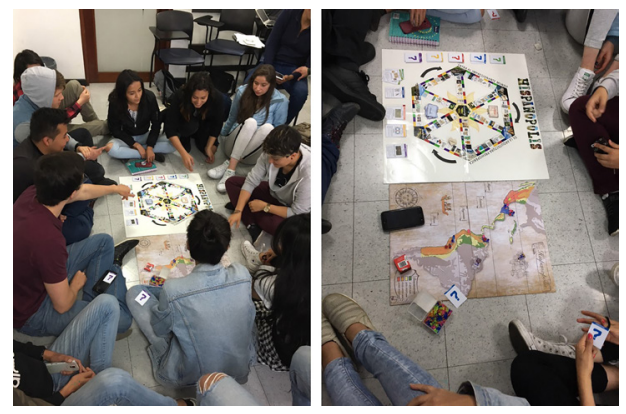

Figura 3: Estudiantes jugando Prehispanópolis Fuente: Elaboración propia 


\subsection{Prototipo "Camino al Sol"}

Para el desarrollo de esta investigación-creación se contó con un equipo conformado por una arquitecta, quien desarrolló toda la parte correspondiente al diseño gráfico, una diseñadora industrial, quien se encargó de la elaboración del empaque (caja y divisiones internas), y además se vinculó, como apoyo a toda la investigación, a un estudiante de arquitectura, quien había participado en la primera versión de Prehispanópolis.

Entre las actividades planteadas se hicieron ajustes de diseño de producto en donde se unificó gráficamente la imagen del juego, y a su vez se realizaron varias pruebas de empaque que se adaptaron al material elaborado y a los estándares de los juegos. Con estos ajustes se procedió a fabricar un primer modelo ajustado, el cua se utilizó para hacer pruebas de usabilidad y validaciones del juego con docentes y estudiantes de arquitectura. Al primer modelo ajustado se le realizaron mejoras en cuanto a diseño, contenido y jugabilidad, para finalmente desarrollar el prototipo de Camino al Sol. Siguiendo en la misma línea, expuesta con anterioridad, se explicarán estos ajustes sobre las dinámicas, mecánicas, componentes y la experiencia.

En cuanto a las dinámicas, refiriéndonos a la narrativa y al aprendizaje, lo primero que se modificó fue el nombre del juego. Se buscaba un nombre que agrupara las cuatro culturas y reforzara la identidad indígena, por lo que se pensó, en ese momento, en cuál elemento era común a todas estas culturas prehispánicas. Se toma al sol como este elemento central, utilizándose para el nombre y como referente de inspiración para e diseño visual. También se unificaron las categorías de preguntas y se ampliaron, incluyendo una nueva ciudad para completar ocho ciudades de la siguiente forma. De la cultura Maya: Palenque, Chichen Itzá y Uxmal; de la cultura Teotihuacana: Teotihuacán; de la cultura Azteca: Tenochtitlán; de la cultura Inca: Cusco y Machu Picchu, y de la cultura Tairona, la ciudad de Teyuna.

\section{Resultado: Jugando Camino al Sol}

El juego consiste en la conformación de un máximo de ocho equipos, cada uno de los cuales representará una ciudad de las mencionadas anteriormente. En el tablero de juego se ubica el tótem de cada ciudad en la casilla correspondiente. Luego, con el número logrado con los dados, se avanza por las casillas. A medida que se contestan las preguntas de manera correcta, se pueden sortear los sacrificios y conseguir ofrendas, con las cuales se obtienen los diferentes elementos que conforman las maquetas de cada ciudad (pirámides, viviendas, caminos, ríos, entre otros). El ganador será el primer jugador o equipo que Ilegue al Sol, completando las maquetas con sus diez elementos.

Los componentes de Camino al Sol son: una guía con las instrucciones del juego, un tablero de juego, ocho tótems (uno por ciudad), 80 tarjetas de preguntas, 20 tarjetas de sacrificio, 20 tarjetas de ofrenda, 20 tarjetas de reto, 80 tarjetas de información, ocho maquetas (una por ciudad) con diez elementos por ciudad y dos dados mayas (Figura 4).

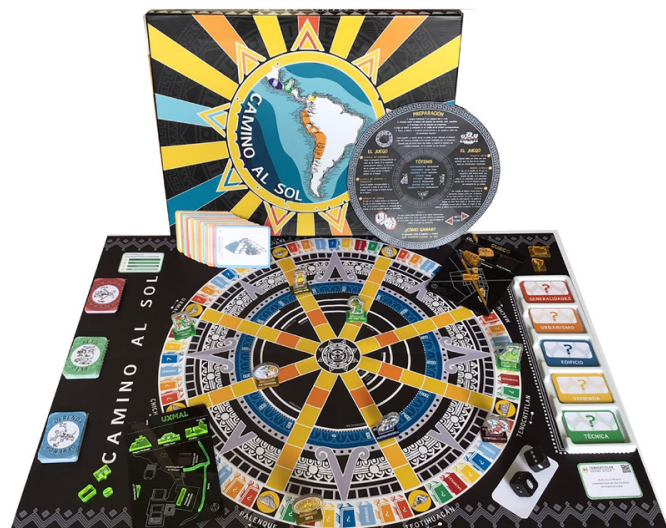

Figura 4: Camino al Sol, componentes

Fuente: Elaboración propia

Para Camino al Sol se elaboraron unos dados con los números utilizados por la cultura maya, la cual tenía el concepto del cero, que era simbolizado en forma de concha o semilla. Los números del uno al cuatro son representados por puntos, mientras el número cinco es representado por una barra horizontal. Al utilizar esta numeración, los estudiantes conocen y valoran los avances de la cultura maya. Los tótems son las fichas con las que se avanza en el tablero y representan a cada una de las ciudades. Para el diseño de tótem se escogió uno de los dioses más representativos de cada ciudad.

Camino al Sol tiene cinco categorías de preguntas: generalidades, urbanismo, edificios, vivienda y técnica. En total Camino al Sol cuenta con ochenta preguntas, dos de cada categoría para cada la ciudad. Cada tarjeta contiene la pregunta, el nombre de la cultura, la ciudad, un número y un código QR (código de respuesta rápida). Para jugar Camino al Sol se debe contar con unos conocimientos previos sobre la arquitectura y el urbanismo de las culturas prehispánicas, aunque la respuesta se puede validar con las tarjetas de información, que a su vez sirven para estudiar fuera del juego. En estas tarjetas se encuentra, por el anverso, el número de la pregunta, el nombre de la cultura, la ciudad y una imagen ilustrativa. Por el reverso de la tarjeta se encuentra el texto resumen de la respuesta a la pregunta (Figura 5).

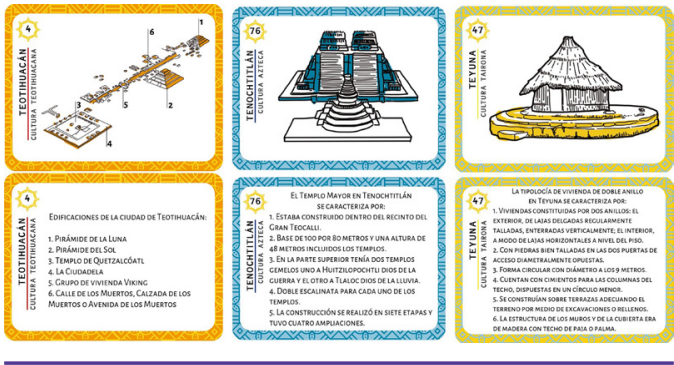

Figura 5: Camino al Sol, tarjetas de información

Fuente: Elaboración propia

En el tablero se encuentran unas casillas de ofrenda y sacrificio, en las cuales se pueden ganar o perder elementos directamente o con el lanzamiento de los dados. Las tarjetas de ofrenda y sacrificio están redactadas de tal forma que se involucran características culturales y dioses, generando una mayor recordación en los estudiantes. Para las tarjetas reto se establecieron tres categorías: una de selección múltiple, con preguntas relacionadas con la localización de las ciudades; 
la segunda categoría es de reto de dibujo, y la tercera está relacionada con adivinar una deidad.

La imagen del tablero está inspirada en el patolli ${ }^{4}$ y en el tonalpohualli ${ }^{5}$. Además de esto, se usaron referentes y colores alusivos a las culturas prehispánicas con textos y símbolos fáciles de leer, incorporando en el diseño de tablero los espacios para ubicar las diferentes tarjetas. Respecto al contenido del tablero, el objetivo es que sirva como material pedagógico. En este sentido se divide el tablero desde el centro hacia los lados, agrupando las ciudades por culturas, y a su vez en el centro del tablero se puede leer una línea de tiempo desde el año 100 A.C. hasta el año 1500 D.C.

Cada ciudad cuenta con una maqueta que tiene la abstracción de la planta urbana y diez elementos representativos que encajan en la ciudad, dos por cada una de las categorías establecidas. Estos elementos son los que se van ganando cada vez que se conteste correctamente o a través de las ofrendas y retos. Estos tableros territorios, además de utilizarse en el juego, se pueden usar para entender y estudiar la estructura urbana y elementos característicos de cada ciudad (Figura 6).

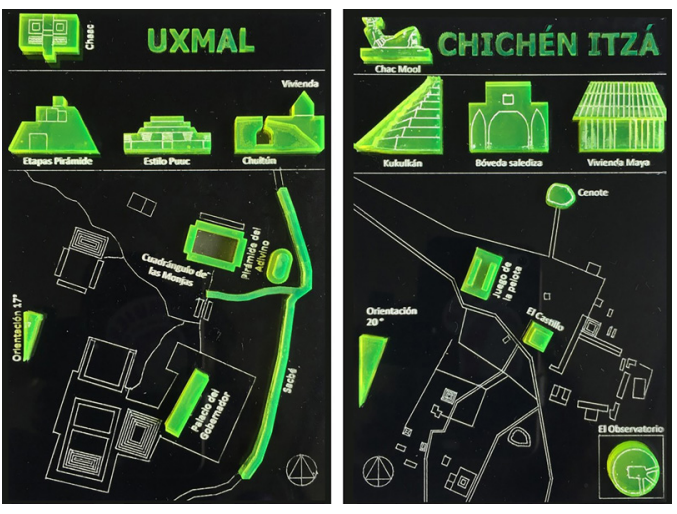

Figura 6: Camino al Sol, maquetas tablero- territorio Fuente: Elaboración propia

Finalmente, el juego se utilizó principalmente como una estrategia de aprendizaje complementaria a las clases magistrales. Una vez finalizadas las exposiciones sobre las ciudades prehispánicas, se procedió a jugar Camino al Sol. Debido a la importancia de este proceso, desarrollamos una sección independiente que se desarrolla a continuación. Esta da cuenta del proceso en el que se obtuvo información de entrevistas, encuestas, grupos focales y análisis de los resultados de las evaluaciones de los estudiantes.

\section{Evaluación de la estrategia pedagógica}

La validación del primer modelo ajustado de Camino al Sol se realizó en el segundo semestre del año 2019. Se jugó con dos grupos de estudiantes de la asignatura Arquitectura Hispanoamericana. Un grupo tenía 21 estudiantes y el otro

${ }^{4}$ El patolli fue un juego de apuestas muy difundido en Mesoamérica. Lo practicaron tehotihuacanos, toltecas, mayas y aztecas.

Calendario sagrado de los aztecas del México precolombino, constituido por 260 días repartidos en trece meses de veinte días cada uno. grupo 23 estudiantes, para un total de 44. Primero, los estudiantes se dividieron por grupos y realizaron exposiciones en clase de cada una de las ciudades prehispánica; luego se jugaba Camino al Sol para reforzar conocimientos y aclarar dudas sobre algún tema específico. En la última clase de este periodo se jugó Camino al Sol (Figura 7), y posterior a esto se aplicó el mismo examen que se hacía en los semestres anteriores para poder comparar si había un mejor desempeño en la prueba.

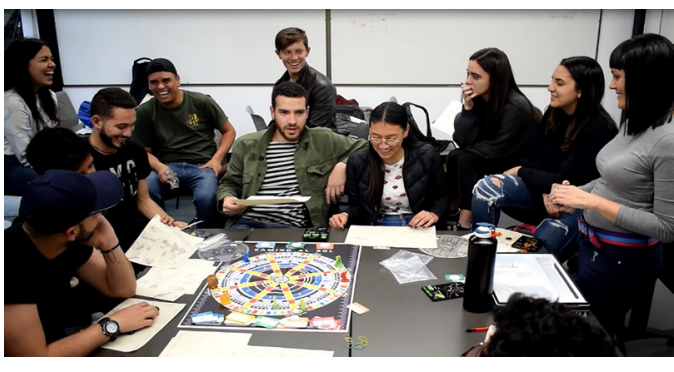

Figura 7: Camino al Sol, experiencia con estudiantes de segundo periodo del 2019

Fuente: Elaboración propia

Paralelamente se realizó una encuesta a los 44 estudiantes, de los cuales respondieron 33. La encuesta se estructuró en los siguientes puntos: experiencia, jugabilidad, diseño, y aprendizaje. Además, se realizaron tres entrevistas en profundidad con estudiantes que representaban diferentes antecedentes de logros académicos. También se realizaron dos grupos focales, moderados por el asistente de investigación, con la presencia de todos los estudiantes de cada grupo sobre los mismos temas. El objetivo era que a través de los grupos focales se capturara información que los estudiantes podrían estar omitiendo, tanto en encuestas como en entrevistas. Aparte se compararon las calificaciones obtenidas por los estudiantes del grupo que utilizó el juego en 2019, con en el mismo examen de los estudiantes de 2017 , quienes no tuvieron esta experiencia.

En cuanto a las encuestas, se realizaron ocho preguntas sobre la experiencia del juego. De las respuestas resaltamos que alrededor del $90 \%$ de los estudiantes consideraron que jugar Camino al Sol fue muy emocionante y animado. 75\% consideraron que el juego les ayudó a relacionarse con sus compañeros, y casi la totalidad (95\%), afirmaron que el juego fomentó su participación en clase. En cuanto a las actividades que les resultaron más divertidas del juego, los estudiantes resaltaron el poner en práctica su conocimiento, el proceso de armado de las maquetas y los retos y recompensas. En cuanto a aspectos negativos, el 20\% de los estudiantes afirmaron que se deben aclarar algunas reglas, y un $12 \%$ afirmaron que el juego duraba mucho tiempo.

En jugabilidad se realizaron cuatro preguntas. El 57\% afirmó que el juego es largo o muy largo reiterando lo concluido en la experiencia, y la gran mayoría de los estudiantes estuvo de acuerdo en que el azar fuera un factor principal para ganar y estuvo conforme con las reglas.

Finalmente, en cuanto a aprendizaje, alrededor del 90\% afirmó que el juego fue una motivación para aprender 
sobre historia y gracias al juego le daban más ganas de asistir a clase. Consideraron que Camino al Sol es útil para reforzar los conocimientos, para recordarlos y, en menor medida, para evaluar. Todos los estudiantes estuvieron de acuerdo en implementar juegos en las otras clases de historia, y especialmente historia de la arquitectura medieval.

Las entrevistas y grupos focales reforzaron los resultados de las encuestas e introdujeron nuevos aspectos. Por ejemplo, los estudiantes afirmaron que el juego les permitió revalorizar la cultura prehispánica. Un estudiante mencionó: "no solamente ayuda a estudiar de una manera más dinámica, sino que también reivindica culturas que uno tiene en el olvido". También los estudiantes afirmaron que el juego no solamente les permite aprender, sino que también les facilita la interacción entre ellos. "Yo creo que uno, mediante el juego, aprende mucho más efectivamente y no solo adquiere conocimientos, sino que también afianza las relaciones sociales entre las personas con las que está jugando", afirmó otro participante. Una de las ideas que se repitieron en las conversaciones fue el valor de juego para crear una motivación efectiva por medio de la competencia entre los grupos, por ejemplo, para adquirir la totalidad de las propiedades de su maqueta. Esto estimula el interés del estudiante por adquirir e conocimiento necesario para ganar, a la vez que hace más práctico y divertido el hecho de estudiar un contenido entendido como monótono. Demostrando esto, otro estudiante dijo: "(El juego) es mucho más efectivo, se queda muy, muy guardado en la memoria, y es muy divertido aprender así, de una manera muy lúdica, que me parece excelente"

Entre otros aspectos se afirmó que la asociación de contenido escrito con el contenido gráfico/visual permite una mayor recordación. "Lo que está escrito se te ve representado en una imagen, y entonces, al asociar la imagen con el texto, queda más fácil", mencionó una estudiante.

Otros mencionaron que la accesibilidad a las tarjetas de preguntas desde cualquier computador, tablet o teléfono inteligente, permitió que el estudiante en clase se concentre en el contenido y no en transcribir la información en su cuaderno, a la vez que sintetiza la información relevante para el curso. Esto, a diferencia de cuando se buscan datos en internet, donde la oferta es tan amplia y generalizada que el estudiante se concentra en información poco relevante para el curso. Finalmente, en las entrevistas y grupos focales los estudiantes también sugirieron que se implementara un modo de juego más rápido, según las necesidades y posibilidades de tiempo por clase.

El último paso de verificación fue la comparación de los resultados del mismo examen escrito aplicado e segundo semestre de 2017 (34 estudiantes), que no utilizaron el juego, y el segundo semestre de 2019 (30 estudiantes), que jugaron Camino al Sol. En 2017 los resultados tuvieron un promedio de 6.5/10 y en 2019 e promedio fue 8.1/10. Entre estos resultados la desviación estándar fue en 2017 de 2.1, y en 2019 de 1.6. Para 2017 la nota mínima fue de 1/10 y para 2019 de 5/10, mientras en ambos periodos la nota máxima fue de
10/10. Los resultados son mejores en 2019, con mejores notas y menor variabilidad entre los resultados de los diferentes estudiantes, lo que puede tomarse, a pesar de sus limitaciones, como un indicio de los beneficios de Camino al Sol como ejemplo positivo del ABJ y de la gamificación.

\section{Conclusiones}

Este artículo resume el proceso de diseño e implementación de una estrategia de $A B J$ y gamificación aplicada a una clase de historia de arquitectura y urbanismo prehispánico para estudiantes universitarios. La estrategia se materializó a través de Camino al Sol, un juego de mesa para mejorar la motivación y el aprendizaje de los estudiantes. De las estrategias del ABJ se resalta diseñar el juego con un objetivo específico de aprendizaje; mejorar la enseñanza a través de la motivación por jugar; y crear un adecuado balance entre dinámicas, mecánicas, componentes para crear una buena experiencia lúdica.

La experiencia se dividió en tres momentos, en el primero se planteó un ejercicio preliminar en el año 2018 llamado Prehispanópolis, un juego de mesa que contó con la participación de los estudiantes en su diseño y fabricación. En la concepción y construcción del juego los estudiantes fortalecieron su conocimiento con el proceso de elaboración y experimentaron cómo se concibe y se materializa una idea para luego ponerla en marcha en la clase. Un segundo momento fue la formulación de un nuevo juego de mesa, en el año 2019, basado en los aprendizajes de la experiencia anterior, en el marco de un proyecto de investigación-creación con el apoyo de dos profesionales en diseño y un asistente de investigación. El nuevo juego, Camino al Sol, se compone de un tablero, unos tótems, tarjetas, maquetas y dados; el objetivo es avanzar en el tablero y completar las maquetas a través de responder preguntas sobre la clase. El último y tercer momento fue la validación de la utilidad del juego para el aprendizaje. Esto se hizo a través de encuestas, entrevistas, grupos focales y comparación de las evaluaciones de los estudiantes.

A través de estos tres momentos demostramos que las estrategias $A B J$, efectivamente motivan a los estudiantes a asistir a clase y a aprender sobre la arquitectura prehispánica, además de experimentar cómo el proyecto de investigación en sí mismo se convierte también en una experiencia de aprendizaje. Esto lo logra a través de la interacción entre los estudiantes. Se encontró que el juego promueve la socialización y el estudio grupal.

El juego, además, genera empatía no solo con el contenido teórico sino entre compañeros, lo que fomenta una mayor participación de cada estudiante en el grupo y un mejor resultado en sus evaluaciones individuales.

Otra de las fortalezas de Camino al Sol fue transmitir e conocimiento a los estudiantes por medio del material que asocia el contenido escrito con un contenido gráfico/visual de alta calidad, que permite una mayor recordación. En cuanto a aspectos que se pueden mejorar, se encontró que el desarrollo del juego podría ser más corto para adaptarse a los tiempos de la clase. 
Sin embargo, consideramos que este estudio, a pesar de sus limitaciones, constituye un pionero para la transformación de los métodos de enseñanza para un entorno cambiante.

\section{Agradecimientos}

Camino al Sol contó con la financiación de la Pontificia Universidad Javeriana, Bogotá, bajo el proyecto de investigación - creación ID PPTA 8500. Nos gustaría agradecer la colaboración de la arquitecta Daniela Siabato Jara, la diseñadora industrial Silvia Patricia Bernal Castro y el estudiante de arquitectura Julián Mauricio Castañeda.

Cómo citar este artículo/How to cite this article: Real-Ramos, Y. y Yunda, J. G.(2021). Aprendizaje basado en el juego aplicado a la enseñanza de la historia de la arquitectura prehispánica. Estoa. Revista de la Facultad de Arquitectura y Urbanismo de la Universidad de Cuenca, 10(19), 67-75. doi: 10.18537/est.v010.n019.a06

\section{Referencias bibliográficas}

Alba Dorado, M. (2018). Estrategias formativas en la iniciación del aprendizaje del proyecto arquitectónico. Estoa. Revista de la Facultad de Arquitectura y Urbanismo de la Universidad de Cuenca, 7(14), 17-29. https://doi. org/10.18537/est.v007.n014.a01

$\mathrm{CAE}+\mathrm{E}$. Centro para el Aprendizaje la Enseñanza y la Evaluación. (2016). Curso de planeación de la enseñanza. Pontificia Universidad Javeriana. https://caee.javeriana. edu.co/documents/3869371/0/documento+caee.pdf/ b96c1a61-9fca-4742-81b2-6177f8904526

Cortizo Pérez, J., Carrero García, F., Monsalve Piqueras, B., Velasco Collado, A., Díaz del Dedo, L. y Pérez Martín, J. (2011). Gamificación y Docencia: Lo que la Universidad tiene que aprender de los Videojuegos. VIII Jornadas de Innovación Universitaria. Retos y oportunidades del desarrollo de los nuevos títulos en educación superior. Universidad Europea de Madrid. http://abacus.universidadeuropea.es/ bitstream/handle/11268/1750/46_Gamificacion. pdf?sequence $=2 \&$ isAllowed $=y$

Hamari, J., Shernoff, D. J., Rowe, E., Coller, B., AsbellClarke, J. y Edwards, T. (2016). Challenging games help students learn: An empirical study on engagement, flow and immersion in game-based learning. Computers in Human Behavior, 54, 170-179. https://doi.org/10.1016/j. chb.2015.07.045

Kiili, K. (2005). Digital game-based learning: Towards an experiential gaming model. The Internet and Higher Education, 8(1), 13-24. https://doi.org/10.1016/j. iheduc.2004.12.001

Lion, C. y Maggio, M. (2019). Desafíos para la enseñanza universitaria en los escenarios digitales contemporáneos.
Aportes desde la investigación. Cuadernos de Investigación Educativa, 10(1), 13 - 25. https://doi. org/10.18861/cied.2019.10.1.2878

Montoya-Álvarez, C. y Uribe-Ciro, D. (2016). Jugar para aprender no es aprender jugando: ludificación de procesos pedagógicos. Revista Perspectiva Empresarial, 3(2), 15-25. https://doi.org/10.16967/rpe.v3n2a3

Nor Azan, M., Azizah, J. y Wong Seng, Y. (2009). Digital Game-based learning (DGBL) model and development methodology for teaching history. WSEAS Transactions on Computers, 8(2), 322-333. https://pdfs.semanticscholar. org/0128/1d0d54d0766964fafb29ccefc0206f11be87. pdf

Oliva, H. (2016). La gamificación como estrategia metodológica en el contexto educativo universitario. Realidad y Reflexión (44), 29-47. https://doi.org/10.5377/ ryr.v44i0.3563

Pisabarro Marrón, A. y Vivaracho Pascual, C. (2018). Gamificación en el aula: gincana de programación. ReVisión, 11(1), 85-93. http://www.aenui.net/ojs/index.

Plass, J. L., Homer, B. D. y Kinzer, C. K. (2015). Foundations of Game-Based Learning. Educational Psychologist, 50(4), 258-283. https://doi.org/10.1080/00461520.201 5.1122533

Pontificia Universidad Javeriana. (s.f.). Syllabus. Asignatura Arquitectura Hispanoamericana.

Prensky, M. (2003). Digital game-based learning Computers in Entertainment, 1(1), 21. https://doi. org/10.1145/950566.950596

Prieto Martin, A., Díaz Martin, D., Monserrat Sanz, J. y Reyes Martín, E. (2014). Experiencias de aplicación de estrategias de gamificación a entornos de aprendizaje universitario. ReVisión, 7(2), 76-92. http://aenui.net/ojs/ index.

Qian, M. y Clark, K. R. (2016). Game-based Learning and 21st century skills: A review of recent research. Computers in Human Behavior, 63, 50-58. https://doi. org/10.1016/j.chb.2016.05.023

Verdú Surroca, N. (2016). El rol de las TIC como un recurso en la enseñanza y aprendizaje de la Historia. Workshop: Insegnare e valorizzare la Storia della Sardegna. Cultura, identità, scuola e innovazione didattica (pp. 1-13). Istituto di Storia dell'Europa Mediterranea - Cnr. https:// repositori.udl.cat/bitstream/handle/10459.1/63153/ nov2016. pdf?sequence $=1 \&$ isAllowed $=y$

Villlalustre Martínez, L. y del Moral Pérez, M. (2015). Gamificación: Estrategia para optimizar el proceso de aprendizaje y la adquisición de competencias en contextos universitarios. Digital Education Review, (27), 13-37. https://doi.org/10.1344/der.2015.27.13-31

Werbach, K. y Hunter, D. (2015). The gamification toolkit: Dynamics, mechanics and components for the win. Warton Digital Press. 